\title{
Estimation of Reliability for Stress-Strength Cascade Model
}

\author{
Rohit R. Mutkekar'1 , Surekha B. Munoli² \\ ${ }^{1}$ Goa Institute of Management, Sanquelim, India \\ ${ }^{2}$ Department of Statistics, Karnatak University, Dharwad, India \\ Email: rohitrm@gim.ac.in, sbmunoli@yahoo.co.in
}

How to cite this paper: Mutkekar, R.R. and Munoli, S.B. (2016) Estimation of Reliability for Stress-Strength Cascade Model. Open Journal of Statistics, 6, 873-881. http://dx.doi.org/10.4236/ojs.2016.65072

Received: March 25, 2016

Accepted: October 18, 2016

Published: October 21, 2016

Copyright $\odot 2016$ by authors and Scientific Research Publishing Inc. This work is licensed under the Creative Commons Attribution International License (CC BY 4.0).

http://creativecommons.org/licenses/by/4.0/

\begin{abstract}
The study endeavors to provide statistical inference for a $(1+1)$ cascade system for exponential distribution under joint effect of stress-strength attenuation factors. Estimators of reliability function are obtained using Maximum Likelihood Estimator (MLE) and Uniformly Minimum Variance Unbiased Estimator (UMVUE) of the parameters. Asymptotic distribution of the parameters is also obtained. Comparison between estimators is made using data obtained through simulation experiment.
\end{abstract}

\section{Keywords}

Cascade System, Strength Attenuation Factor, Reliability Modelling, Life Testing Experiment, Estimators of Reliability Function

\section{(c) (i) Open Access}

\section{Introduction}

As the complexity of a system increases, its reliability decreases unless compensatory measures are taken. System reliability can be increased by increasing the reliability of its associated components, but sometimes this cannot be achieved beyond certain limits. An alternative way to increase the reliability in such situation is to have redundant configuration of components in the system.

Cascade system is one such special type of standby system. Cascade redundancy is a hierarchical standby redundancy, where an array of components (finite in number) are arranged in the order of activation. Here, the first component is active and the remaining components are at standby. The brunt of attack, in the first instance is borne by the active component. If it survives the attack, the system also survives with no loss and is ready to face the next attack. However, if the active component fails then the next component in the array has to face and withstand the "cushioned" attack on it. The 
stress acting on the subsequent active component will be " $k$ " times the stress of the previous failed components, where " $k$ " denotes stress attenuation factor.

Research works on reliability modelling and assessment related to cascade model as studied in the literature are quite exhaustive, Pandit and Sriwastav (1975) have featured relevance of geometric distribution in the study of behavior of a cascade system [1]. Raghavachar, Rao and Ramacharyulu (1983) presented a closed form solution of stress attenuated reliability function for $\mathrm{n}$-cascade system when both stress and strength follow identical distributions [2]. Maheshwari, Rekha, Rao and Raghavachar (1993) studied stress attenuated reliability for $\mathrm{n}$-cascade system whose stress and strength follow normal and exponential distributions respectively [3]. Rekha and Shyam Sunder (1997) have also highlighted a similar cascade system where stress and strength follow gamma and exponential distributions respectively. They showed that for higher parametric values and lower attenuation factors a high degree of reliability could be attained [4]. Rekha and ChechuRaju (1999) endeavored to present a closed form solution of stress attenuated reliability function for $\mathrm{n}$-cascade system with exponential stress and standby strengths following Rayleigh and exponential distributions [5]. Shyam Sunder (2012) has studied stress attenuation for cascade system when both stress and strength follow Rayleigh distribution [6]. In most of the works mentioned in the literature on cascade model, study is carried out by considering the influence of stress attenuation factor only. This observation has motivated the present study of attempting to design reliability model for a cascade system under joint effect of stress as well as strength attenuation factors. Further, reliability assessment (estimation of reliability function) is carried using the standard methods [7]-[10].

\section{Estimation of Reliability for a $(1+1)$ Cascade Model}

\subsection{Stress-Strength Cascade Model}

Let $X_{1}, X_{2}, \cdots, X_{n}$ denote the strengths of $\mathrm{n}$-components in the order of activation and let $Y_{1}, Y_{2}, \cdots, Y_{n}$ be the corresponding stresses acting on them. In a n-cascade system after every failure the stress gets modified by a factor " $k$ " (stress attenuation factor) such that $Y_{2}=k Y_{1}, Y_{3}=k Y_{2}=k^{2} Y_{1}, \cdots, Y_{i}=k^{i-1} Y_{1}$ here, $k>1$ and we assume that the strength gets modified by a factor " $m$ " (strength attenuation factor) such that

$X_{2}=m X_{1}, X_{3}=m X_{2}=m^{2} X_{1}, \cdots, X_{i}=m^{i-1} X_{1}$ here, $0<m<1$.

The reliability function $R_{n}$ of the system with ' $n$ ' components is defined as,

$$
R_{n}=R(1)+R(2)+\cdots+R(n)
$$

where,

$$
R(r)=P\left[X_{1}<Y_{1}, m X_{1}<k Y_{1}, \cdots, m^{r-2} X_{1}<k^{r-2} Y_{1}, m^{r-1} X_{1} \geq k^{r-1} Y_{1}\right] \text { for } r=1,2, \cdots, n
$$

Cascade model with more number of standby components is not recommended as the strength goes on depleting with the order of standby which leads to dead investment. In view of this fact, we have considered estimation of reliability for a $(1+1)$ cascade model. 


\subsection{Reliability Function for a $(1+1)$ Cascade Model}

To determine reliability function for the model under study, let us consider the strength of the two components (basic and standby) to be $X_{1}$ and $X_{2}$ respectively, where $X_{1}, X_{2}$ are independently and identically distributed (i.i.d) exponential random variables with parameter " $\lambda$ ". Let $Y_{1}$ and $Y_{2}$ be the stress acting on the two components respectively, where $Y_{1}, Y_{2}$ are i.i.d exponential random variables with parameter ' $\mu$ '. To obtain the expression for reliability function, consider,

$$
\begin{aligned}
R(1) & =P\left[X_{1} \geq Y_{1}\right]=\int_{0}^{\infty} P\left[X_{1} \geq y_{1}\right] g_{Y_{1}}\left(y_{1}\right) \mathrm{d} y_{1}=\frac{\mu}{(\lambda+\mu)} \\
R(2) & =P\left[X_{1}<Y_{1}, X_{2} \geq Y_{2}\right]=P\left[X_{1}<Y_{1}, m X_{1} \geq k Y_{1}\right] \\
& =\int_{0}^{\infty} P\left[X_{1}<y_{1}\right] P\left[m X_{1} \geq k y_{1}\right] g_{Y_{1}}\left(y_{1}\right) \mathrm{d} y_{1} \\
& =\frac{\mu \lambda}{\left(\mu+\lambda \frac{k}{m}\right)\left\{\mu+\lambda\left(\frac{k}{m}+1\right)\right\}}
\end{aligned}
$$

Using results of (1) and (2), we obtain reliability function for the proposed $(1+1)$ cascade model as,

$$
R_{2}=R(1)+R(2)=\frac{\mu}{(\lambda+\mu)}+\frac{\mu \lambda}{\left(\mu+\lambda \frac{k}{m}\right)\left\{\mu+\lambda\left(\frac{k}{m}+1\right)\right\}}
$$

\subsection{Life Testing Experiment}

To obtain the estimators of " $R_{2}$ ", suppose " $n$ " systems whose reliability function is defined as in expression (3) are put on life testing experiment. Here, $X_{1 i}, X_{2 i}(i=1,2, \cdots, n)$ are observed and $X_{1 i}, X_{2 i}$ are i.i.d exponential random variables with parameters " $\lambda$ " and " $\frac{\lambda}{m}$ ” respectively. Also, the data of stress $Y_{1 i}, Y_{2 i}(i=1,2, \cdots, n)$ are obtained separately from simulation of conditions of the operating environment and $Y_{1 i}, Y_{2 i}$ are i.i.d exponential random variables with parameter " $\mu$ " and " $\frac{\mu}{k}$ ” respectively. The joint probability density function of the random variables $X_{1 i}, X_{2 i}(i=1,2, \cdots, n)$ and $Y_{1 i}, Y_{2 i}(i=1,2, \cdots, n)$ is given by,

$$
\begin{aligned}
L & =\prod_{i=1}^{n}\left[\left(\lambda \mathrm{e}^{-\lambda x_{1 i}}\right)\left(\frac{\lambda}{m} \mathrm{e}^{-\frac{\lambda}{m} x_{2 i}}\right)\right] \prod_{i=1}^{n}\left[\left(\mu \mathrm{e}^{-\mu y_{1 i}}\right)\left(\frac{\mu}{k} \mathrm{e}^{-\frac{\mu}{k} y_{2 i}}\right)\right] \\
& =\frac{\lambda^{2 n}}{m^{n}} \mathrm{e}^{-\lambda\left[\sum_{i=1}^{n} x_{1 i}+\frac{1}{m} \sum_{i=1}^{n} x_{2 i}\right]} \frac{\mu^{2 n}}{k^{n}} \mathrm{e}^{-\mu\left[\sum_{i=1}^{n} y_{1 i}+\frac{1}{k} \sum_{i=1}^{n} y_{2 i}\right]} \\
& =\frac{\lambda^{2 n}}{m^{n}} \mathrm{e}^{-\lambda\left[x_{1}^{\prime}+\frac{x_{2}^{\prime}}{m}\right]} \frac{\mu^{2 n}}{k^{n}} \mathrm{e}^{-\mu\left[y_{1}^{\prime}+\frac{y_{2}^{\prime}}{k}\right]}
\end{aligned}
$$

where,

$$
x_{1}^{\prime}=\sum_{i=1}^{n} x_{1 i}, \quad x_{2}^{\prime}=\sum_{i=1}^{n} x_{2 i}, \quad y_{1}^{\prime}=\sum_{i=1}^{n} y_{1 i}, \quad y_{2}^{\prime}=\sum_{i=1}^{n} y_{2 i}
$$


The log-likelihood function of Equation (4) is obtained as,

$$
\log L=2 n \log \lambda-n \log m-\lambda\left[x_{1}^{\prime}+\frac{x_{2}^{\prime}}{m}\right]+2 n \log \mu-n \log k-\mu\left[y_{1}^{\prime}+\frac{y_{2}^{\prime}}{k}\right]
$$

\subsection{Estimators of Reliability Function (MLE \& UMVUE)}

Differentiating the log-likelihood function given in Equation (5) partially with respect to $\lambda, m$ and equating it to zero, we get,

$$
\begin{aligned}
& \frac{\partial \log L}{\partial \lambda}=0 \Rightarrow \frac{2 n}{\lambda}-\left[x_{1}^{\prime}+\frac{x_{2}^{\prime}}{m}\right]=0 \\
& \frac{\partial \log L}{\partial m}=0 \Rightarrow \frac{-n}{m}+\frac{\lambda x_{2}^{\prime}}{m^{2}}=0 \Rightarrow-n+\frac{\lambda x_{2}^{\prime}}{m}=0
\end{aligned}
$$

Solving Equations ((6) and (7)) simultaneously, we get the Maximum Likelihood Estimator (MLE) of $\lambda$ and $m$ as,

$$
\begin{aligned}
& \hat{\lambda}=\frac{n}{x_{1}^{\prime}} \\
& \hat{m}=\frac{x_{2}^{\prime}}{x_{1}^{\prime}}
\end{aligned}
$$

Similarly, differentiating the log-likelihood function given in Equation (5) with respect to $\mu, k$ and equating it to zero, we get,

$$
\begin{aligned}
& \frac{\partial \log L}{\partial \mu}=0 \Rightarrow \frac{2 n}{\mu}-\left[y_{1}^{\prime}+\frac{y_{2}^{\prime}}{k}\right]=0 \\
& \frac{\partial \log L}{\partial k}=0 \Rightarrow \frac{-n}{k}+\frac{\mu y_{2}^{\prime}}{k^{2}}=0 \Rightarrow-n+\frac{\mu y_{2}^{\prime}}{k}=0
\end{aligned}
$$

Solving Equations ((10) and (11)) simultaneously, we get the MLE of $\mu$ and $k$ as,

$$
\begin{gathered}
\hat{\mu}=\frac{n}{y_{1}^{\prime}} \\
\hat{k}=\frac{y_{2}^{\prime}}{y_{1}^{\prime}}
\end{gathered}
$$

Using the invariance property of MLE, the MLE of reliability function ' $\widehat{R_{2}}$ ' is obtained by substituting the MLEs of $\lambda, m, \mu, k$ in Equation (3) and is given by,

$$
\widehat{R_{2}}=\frac{\hat{\mu}}{(\hat{\lambda}+\hat{\mu})}+\frac{\hat{\mu} \hat{\lambda}}{\left(\hat{\mu}+\hat{\lambda} \frac{\hat{k}}{\hat{m}}\right)\left\{\hat{\mu}+\hat{\lambda}\left(\frac{\hat{k}}{\hat{m}}+1\right)\right\}}
$$

Here, $\widehat{R_{2}}$ denotes the estimator of reliability function obtained through MLE of the parameters. Further, estimator of the reliability function " $R_{2}^{*}$ " attained through the Uniformly Minimum Variance Unbiased Estimator (UMVUE) of the parameters is obtained as follows.

We know that, $x_{1 i} \sim \operatorname{Exponential}(\lambda)$, implies $x_{1}^{\prime}=\sum_{i=1}^{n} x_{1 i} \sim \operatorname{Gamma}(n, \lambda)$ 


$$
\begin{aligned}
& \Rightarrow E\left[\frac{1}{x_{1}^{\prime}}\right]=\int_{0}^{\infty} \frac{1}{x_{1}^{\prime}} \frac{\lambda^{n} \mathrm{e}^{-\lambda x_{1}^{\prime}}\left(x_{1}^{\prime}\right)^{n-1}}{\Gamma n} \mathrm{~d} x_{1}^{\prime}=\frac{\lambda^{n}}{\Gamma n} \int_{0}^{\infty} \mathrm{e}^{-\lambda x_{1}^{\prime}}\left(x_{1}^{\prime}\right)^{(n-1)-1} \mathrm{~d} x_{1}^{\prime} \\
& \Rightarrow E\left[\frac{(n-1)}{x_{1}^{\prime}}\right]=\lambda \\
& \Rightarrow \lambda^{*}=\left[\frac{(n-1)}{x_{1}^{\prime}}\right] \text { is the UMVUE of parameter } \lambda
\end{aligned}
$$

Also, $y_{1 i} \sim \operatorname{Exponential}(\mu)$, implies $y_{1}^{\prime}=\sum_{i=1}^{n} y_{1 i} \sim \operatorname{Gamma}(n, \mu)$

On similar grounds we have,

$$
\mu^{*}=\left[\frac{(n-1)}{y_{1}^{\prime}}\right] \text { is the UMVUE of parameter } \mu
$$

Similarly, $x_{2 i} \sim$ Exponential $\left(\frac{\lambda}{m}\right)$, implies $x_{2}^{\prime}=\sum_{i=1}^{n} x_{2 i} \sim \operatorname{Gamma}\left(n, \frac{\lambda}{m}\right)$

$$
\begin{aligned}
& \Rightarrow E\left[x_{2}^{\prime}\right]=\int_{0}^{\infty} x_{2}^{\prime} \frac{\left(\frac{\lambda}{m}\right)^{n} \mathrm{e}^{-\frac{\lambda}{m} x_{2}^{\prime}}\left(x_{2}^{\prime}\right)^{n-1}}{\Gamma n} \mathrm{~d} x_{2}^{\prime}=\frac{\left(\frac{\lambda}{m}\right)^{n}}{\Gamma n} \int_{0}^{\infty} \mathrm{e}^{-\frac{\lambda}{m} x_{2}^{\prime}}\left(x_{2}^{\prime}\right)^{(n+1)-1} \mathrm{~d} x_{2}^{\prime} \\
& \Rightarrow E\left[x_{2}^{\prime}\right]=\frac{m n}{\lambda}
\end{aligned}
$$

Substituting $\lambda=\lambda^{*}$ [result as mentioned in Equation (15)], we get,

$$
\begin{aligned}
& \Rightarrow E\left[x_{2}^{\prime}\right]=\frac{m n x_{1}^{\prime}}{n-1} \\
& \Rightarrow E\left[\frac{(n-1) x_{2}^{\prime}}{n x_{1}^{\prime}}\right]=m \\
& \Rightarrow m^{*}=\left[\frac{(n-1) x_{2}^{\prime}}{n x_{1}^{\prime}}\right] \text { is the UMVUE of parameter } m
\end{aligned}
$$

Also, $y_{2 i} \sim$ Exponential $\left(\frac{\mu}{k}\right)$, implies $y_{2}^{\prime}=\sum_{i=1}^{n} y_{2 i} \sim \operatorname{Gamma}\left(n, \frac{\mu}{k}\right)$

On similar grounds we have,

$$
k^{*}=\left[\frac{(n-1) y_{2}^{\prime}}{n y_{1}^{\prime}}\right] \text { is the UMVUE of parameter } k
$$

Substituting the UMVUEs of $\lambda, m, \mu, k$ in Equation (3), we get estimator of the reliability function " $R_{2}^{*}$ ” obtained through the UMVUE of the parameters.

\subsection{Asymptotic Distribution}

To obtain the asymptotic distribution of $\hat{\lambda}, \hat{m}, \hat{\mu}, \hat{k}$, let us denote the Fisher Information Matrix of $\lambda, m, \mu, k$ as $I(\lambda, m, \mu, k)$. 


$$
\begin{aligned}
I(\lambda, m, \mu, k)= & -\left[\begin{array}{llll}
E\left(\frac{\partial^{2} L}{\partial \lambda^{2}}\right) & E\left(\frac{\partial^{2} L}{\partial \lambda \partial m}\right) & E\left(\frac{\partial^{2} L}{\partial \lambda \partial \mu}\right) & E\left(\frac{\partial^{2} L}{\partial \lambda \partial k}\right) \\
E\left(\frac{\partial^{2} L}{\partial m \partial \lambda}\right) & E\left(\frac{\partial^{2} L}{\partial m^{2}}\right) & E\left(\frac{\partial^{2} L}{\partial m \partial \mu}\right) & E\left(\frac{\partial^{2} L}{\partial m \partial k}\right) \\
E\left(\frac{\partial^{2} L}{\partial \mu \partial \lambda}\right) & E\left(\frac{\partial^{2} L}{\partial \mu \partial m}\right) & E\left(\frac{\partial^{2} L}{\partial \mu^{2}}\right) & E\left(\frac{\partial^{2} L}{\partial \mu \partial k}\right) \\
E\left(\frac{\partial^{2} L}{\partial k \partial \lambda}\right) & E\left(\frac{\partial^{2} L}{\partial k \partial m}\right) & E\left(\frac{\partial^{2} L}{\partial k \partial \mu}\right) & E\left(\frac{\partial^{2} L}{\partial k^{2}}\right)
\end{array}\right] \\
= & {\left[\begin{array}{llll}
I_{11} & I_{12} & I_{13} & I_{14} \\
I_{21} & I_{22} & I_{23} & I_{24} \\
I_{31} & I_{32} & I_{33} & I_{34} \\
I_{41} & I_{42} & I_{43} & I_{44}
\end{array}\right] \text { (say) } }
\end{aligned}
$$

where,

$$
\begin{aligned}
I_{11} & =-\left[E\left(\frac{\partial^{2} L}{\partial \lambda^{2}}\right)\right]=\frac{2 n}{\lambda^{2}} \\
I_{22} & =-\left[E\left(\frac{\partial^{2} L}{\partial m^{2}}\right)\right]=\frac{n}{m^{2}} \\
I_{33} & =-\left[E\left(\frac{\partial^{2} L}{\partial \mu^{2}}\right)\right]=\frac{2 n}{\mu^{2}} \\
I_{44} & =-\left[E\left(\frac{\partial^{2} L}{\partial k^{2}}\right)\right]=\frac{n}{k^{2}} \\
-E\left(\frac{\partial^{2} L}{\partial \lambda \partial m}\right) & =-E\left(\frac{\partial^{2} L}{\partial m \partial \lambda}\right) \Rightarrow I_{12}=I_{21}=\frac{-n}{m \lambda} \\
-E\left(\frac{\partial^{2} L}{\partial \mu \partial k}\right) & =-E\left(\frac{\partial^{2} L}{\partial k \partial \mu}\right) \Rightarrow I_{34}=I_{43}=\frac{-n}{k \mu} \\
-E\left(\frac{\partial^{2} L}{\partial \lambda \partial \mu}\right) & =-E\left(\frac{\partial^{2} L}{\partial \mu \partial \lambda}\right)=0 \Rightarrow I_{13}=I_{31}=0 \\
-E\left(\frac{\partial^{2} L}{\partial \lambda \partial k}\right) & =-E\left(\frac{\partial^{2} L}{\partial k \partial \lambda}\right)=0 \Rightarrow I_{14}=I_{41}=0 \\
-E\left(\frac{\partial^{2} L}{\partial m \partial \mu}\right) & =-E\left(\frac{\partial^{2} L}{\partial \mu \partial \partial m}\right)=0 \Rightarrow I_{23}=I_{32}=0 \\
& =-E\left(\frac{\partial^{2} L}{\partial k \partial m}\right)=0 \Rightarrow I_{24}=I_{42}=0
\end{aligned}
$$

Thus, we have the Fisher Information Matrix as, 


$$
I(\lambda, m, \mu, k)=\left[\begin{array}{cccc}
\frac{2 n}{\lambda^{2}} & \frac{-n}{m \lambda} & 0 & 0 \\
\frac{-n}{m \lambda} & \frac{n}{m^{2}} & 0 & 0 \\
0 & 0 & \frac{2 n}{\mu^{2}} & \frac{-n}{k \mu} \\
0 & 0 & \frac{-n}{k \mu} & \frac{n}{k^{2}}
\end{array}\right]
$$

From the asymptotic properties of MLE under regularity conditions and multivariate central limit theorem we have,

$$
[(\hat{\lambda}-\lambda),(\hat{m}-m),(\hat{\mu}-\mu),(\hat{k}-k)] \rightarrow \operatorname{Normal}\left(0, I^{-1}\right)
$$

$I^{-1}$ is inverse of Fisher Information Matrix “ $I(\lambda, m, \mu, k)$ ” where,

$$
I^{-1}=\left[\begin{array}{cccc}
\frac{\lambda^{2}}{n} & \frac{m \lambda}{n} & 0 & 0 \\
\frac{m \lambda}{n} & \frac{2 m^{2}}{n} & 0 & 0 \\
0 & 0 & \frac{\mu^{2}}{n} & \frac{k \mu}{n} \\
0 & 0 & \frac{k \mu}{n} & \frac{2 k^{2}}{n}
\end{array}\right]
$$

\section{Simulation Experiment}

For the $i^{\text {th }}$ system, the random variables $x_{1 i}, x_{2 i}$ (with respect to strength) and random variables $y_{i 1}, y_{i 2}$ (with respect to stress) are generated independently as follows:

Step 1: Initialize $i_{1}=1, n=n_{0}, \lambda=\lambda_{0}, m=m_{0}$ for the $1^{\text {st }}$ and $2^{\text {nd }}$ component of the system. Uniform random numbers $U_{1}\left[i_{1}\right]$ is generated from $U(0,1)$. Further, exponential random variable $x_{1 i}=\left(\frac{-1}{\lambda_{0}}\right) \log \left(1-U_{1}\left[i_{1}\right]\right)$ is obtained for the $1^{\text {st }}$ component of the $i^{\text {th }}$ system. Another uniform random numbers $U_{2}\left[i_{1}\right]$ is generated from $U(0,1)$. Further, exponential random variable $x_{2 i}=\left(\frac{-m_{0}}{\lambda_{0}}\right) \log \left(1-U_{2}\left[i_{1}\right]\right)$ is obtained for the $2^{\text {nd }}$ component of the $i^{\text {th }}$ system.

Step 2: The whole procedure in Step 1 is repeated for $n=n_{0}$ number of systems and the statistics $x_{1}^{\prime}=\sum_{i=1}^{n} x_{1 i}, x_{2}^{\prime}=\sum_{i=1}^{n} x_{2 i}$ are obtained.

Step 3: Initialize $i_{2}=1, n=n_{0}, \mu=\mu_{0}, k=k_{0}$ for the $1^{\text {st }}$ and $2^{\text {nd }}$ component of the system. Uniform random numbers $V_{1}\left[i_{2}\right]$ are generated from $U(0,1)$. Further, exponential random variable $y_{1 i}=\left(\frac{-1}{\mu_{0}}\right) \log \left(1-V_{1}\left[i_{2}\right]\right)$ is obtained for the $1^{\text {st }}$ component of the $i^{\text {th }}$ system. Another uniform random numbers $V_{2}\left[i_{2}\right]$ are generated from 
$U(0,1)$. Further, exponential random variable $y_{2 i}=\left(\frac{-k_{0}}{\mu_{0}}\right) \log \left(1-V_{2}\left[i_{2}\right]\right)$ is obtained for the $2^{\text {nd }}$ component of the $i^{\text {th }}$ system.

Step 4: The whole procedure in Step 3 is repeated for $n=n_{0}$ number of systems and the statistics $y_{1}^{\prime}=\sum_{i=1}^{n} y_{1 i}, y_{2}^{\prime}=\sum_{i=1}^{n} y_{2 i}$ are obtained.

Step 5: With the help of the statistics $x_{1}^{\prime}, x_{2}^{\prime}, y_{1}^{\prime}$ and $y_{2}^{\prime}$ the MLE of parameters $\lambda, m, \mu, k$ of the model are obtained. Using these MLEs in the expression of reliability function, the MLE of reliability function is obtained.

Step 6: With the help of the statistics $x_{1}^{\prime}, x_{2}^{\prime}, y_{1}^{\prime}$ and $y_{2}^{\prime}$ the UMVUE of parameters $\lambda, m, \mu, k$ are obtained. Using these UMVUEs in the expression of reliability function, estimator of the reliability function based on UMVUE of the parameters is obtained.

Table 1 and Table 2 give the results of the above simulation experiment for different values of $\lambda, m, \mu, k$ and $n$.

\section{Conclusion}

From the above results (as shown in Table 1 and Table 2), we observe that reliability of

Table 1. $\lambda=0.5, \mu=1.0, n=5$.

\begin{tabular}{|c|c|c|c|c|c|c|c|c|c|c|c|}
\hline$m$ & $k$ & $R$ & $x_{1}^{\prime}$ & $x_{2}^{\prime}$ & $y_{1}^{\prime}$ & $y_{2}^{\prime}$ & $\hat{R} \quad$ MLE & $\hat{R}$ & UMVUE & MSE MLE & MSE UMVUE \\
\hline 0.25 & 1.25 & 0.792 & 10.290507 & 2.620377 & 5.145254 & 3.800942 & 0.831 & & 0.799 & $1.52 \times 10^{-3}$ & $4.90 \times 10^{-5}$ \\
\hline 0.25 & 1.50 & 0.778 & 09.181533 & 2.998768 & 5.590766 & 3.368335 & 0.816 & & 0.784 & $1.44 \times 10^{-3}$ & $3.60 \times 10^{-5}$ \\
\hline 0.50 & 1.00 & 0.867 & 09.778324 & 3.125278 & 4.889162 & 1.915278 & 0.909 & & 0.860 & $1.76 \times 10^{-3}$ & $4.90 \times 10^{-5}$ \\
\hline 0.50 & 1.50 & 0.833 & 12.241618 & 9.066720 & 6.120809 & 3.797080 & 0.868 & & 0.837 & $1.23 \times 10^{-3}$ & $1.60 \times 10^{-5}$ \\
\hline 0.75 & 1.00 & 0.897 & 14.691534 & 6.720832 & 5.345767 & 2.380555 & 0.931 & & 0.892 & $1.16 \times 10^{-3}$ & $2.50 \times 10^{-5}$ \\
\hline 0.75 & 1.25 & 0.881 & 13.931526 & 7.937316 & 5.965763 & 1.581096 & 0.913 & & 0.877 & $1.03 \times 10^{-3}$ & $1.60 \times 10^{-5}$ \\
\hline 0.75 & 1.50 & 0.867 & 15.716419 & 6.075062 & 6.428210 & 1.075062 & 0.898 & & 0.870 & $9.61 \times 10^{-4}$ & $9.00 \times 10^{-6}$ \\
\hline
\end{tabular}

Table 2. $\lambda=0.5, \mu=1.0, n=10$.

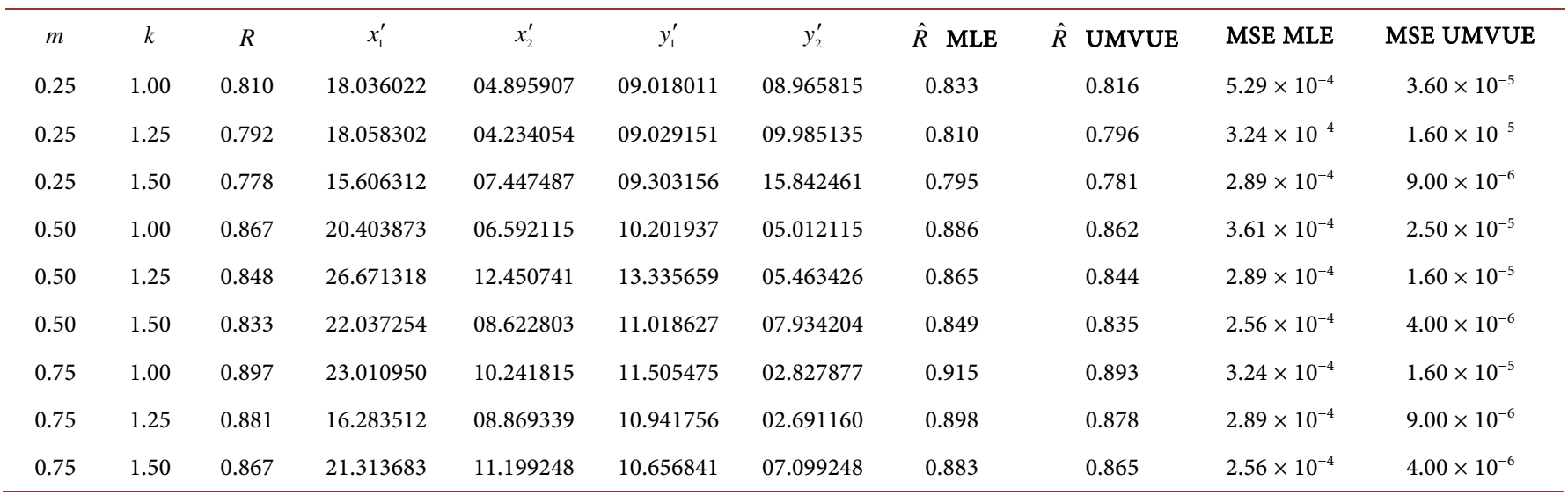


the system improves for larger values of strength attenuation factor $(m)$ and for lower values of stress attenuation factor $(k)$. Here, we also observed the estimates of reliability improves for larger value of the sample size " $n$ ". This indicates that reliability of a system can be enhanced by strengthening the inbuilt mechanism of the system, which ultimately withstands the effects of the external environment in which it operates.

Further, on comparing the efficiencies of MLE of reliability function with reliability estimator obtained using UMVUEs of the parameters, we observed reliability estimator obtained from the UMVUEs of the perform better than the MLE of reliability function in terms of Mean Square Error (MSE) for the given data set. This emphasizes the need to strengthen the processes such that they are least affected by effects of the variation factors which intern boost the reliability of the operating system.

\section{References}

[1] Pandit, S.N.N. and Sriwastav, G.L. (1975) Studies in Cascade Reliability-1.IEEE Transactions on Reliability, R-24, 53-57. http://dx.doi.org/10.1109/TR.1975.5215330

[2] Raghavachar, A.C.N., Kesava Rao, B. and Ramacharyulu Pattabhi, N.C. (1983) Survival Function under Stress Attenuation in Cascade Reliability. Opsearch, 20, 190-207.

[3] Maheshwari, U., Rekha, T.S., Anjan Rao, E. and Raghava Char, A.C.N. (1993) Reliability of a Cascade System with Normal Stress and Exponential Strength. Microelectronics Reliability, 33, 929-936. http://dx.doi.org/10.1016/0026-2714(93)90289-B

[4] Rekha, A. and Shyam Sundar, T. (1997) Reliability of a Cascade System with Exponential Strength and Gamma Stress. Microelectronics Reliability, 37, 683-685. http://dx.doi.org/10.1016/S0026-2714(97)87650-0

[5] Rekha, A. and Chenchu Raju, V.C. (1999) Cascade System Reliability with Rayleigh Distribution. Botswana Journal of Technology, 8, 14-19.

[6] Shyam Sundar, T. (2012) Case Study of Cascade Reliability with Rayleigh Distribution. International Journal of Computer Technology \& Electronics Engineering, 2, 78-87.

[7] Hanagal, D.D. (1996) Estimation of System Reliability in a Two Component Stress-Strength Models. Economic Quality Control, 11, 145-154.

[8] Kunchur, S.H. and Munoli, S.B. (1994) Estimation of Reliability for a Multi Component Survival Stress-Strength Model Based on Exponential Distribution. Communications in Statistics-Theory \& Methods, 23, 3273-3283. http://dx.doi.org/10.1080/03610929408831446

[9] Kundu, D. and Gupta, R.D. (2005) Estimation of $\mathrm{P}(\mathrm{Y}<\mathrm{X})$ for Generalized Exponential Distribution. Metrika, 61, 291-308. http://dx.doi.org/10.1007/s001840400345

[10] Munoli, S.B. and Mutkekar, R.R. (2011) Estimation of Reliability for a Two Component Survival Stress-Strength Model. International Journal of Quality, Statistics \& Reliability, 2011, Article ID: 721962. http://dx.doi.org/10.1155/2011/721962 
Submit or recommend next manuscript to SCIRP and we will provide best service for you:

Accepting pre-submission inquiries through Email, Facebook, LinkedIn, Twitter, etc. A wide selection of journals (inclusive of 9 subjects, more than 200 journals)

Providing 24-hour high-quality service

User-friendly online submission system

Fair and swift peer-review system

Efficient typesetting and proofreading procedure

Display of the result of downloads and visits, as well as the number of cited articles

Maximum dissemination of your research work

Submit your manuscript at: http://papersubmission.scirp.org/

Or contact ojs@scirp.org 